\title{
HIGHLIGHTS
}

OBESITY

\section{Lifestyle counseling ineffective in tackling childhood obesity}

National policies in several countries, including the USA, UK and Australia, endorse the role of general practitioners in monitoring childhood obesity and undertaking any necessary counseling. However, Melissa Wake and colleagues suggest that these costly primary care guidelines are surplus to requirements, as surveillance followed by brief counseling did not improve BMI, nutrition or

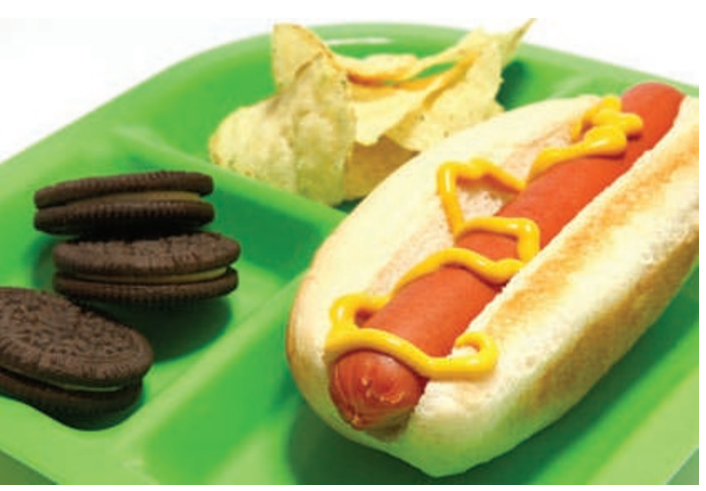

physical activity in children classed as overweight or mildly obese in their study. "Because of the sheer scale of the problem, tackling childhood obesity in the secondary and tertiary health-care sector is not feasible for any but those who are most severely affected," say the authors.

The researchers recruited 66 general practitioners from family practices in Melbourne, Australia, to monitor changes in BMI, nutrition, physical activity and sedentary behavior in 258 children (from more than 4,000 screened) who were overweight or mildly obese according to International Obesity Taskforce criteria. The investigators, involved in the Live Eat And Play (LEAP) 2 trial, randomly assigned the children to attend either four consultations over 12 weeks or no intervention. Crucially, Wake's team report that periodic counseling from a general practitioner had no significant effect on any of the outcomes compared with those in controls. Another important finding was that implementation of the surveillance and counseling program could be approximately 5-15 times more expensive than not intervening at all.

These results corroborate the findings from two smaller trials that used a similar methodology, including the initial LEAP trial, and highlight inefficiencies in current government-proposed strategies to prevent childhood obesity. "Resources may be better divided between primary prevention at the community and population levels, and enhancement of clinical treatment options for children with established obesity," conclude Wake et al.

\section{Rowan Higgs}

Original article Wake, M. et al. Outcomes and costs of primary care surveillance and intervention for overweight or obese children: the LEAP 2 randomised controlled trial. BMJ doi:10.1136/bmj.b3308 